\title{
Generalization and Discovery by Assuming Conserved Mechanisms: Cross-Species Research on Circadian Oscillators
}

\author{
William Bechtel $\$$
}

\begin{abstract}
In many domains of biology, explanation takes the form of characterizing the mechanism responsible for a particular phenomenon in a specific biological system. How are such explanations generalized? One important strategy assumes conservation of mechanisms through evolutionary descent. But conservation is seldom complete. In the case discussed, the central mechanism for circadian rhythms in animals was first identified in Drosophila and then extended to mammals. Scientists' working assumption that the clock mechanisms would be conserved both yielded important generalizations and served as a heuristic for discovery, especially when significant differences between the insect and mammalian mechanism were identified.
\end{abstract}

1. Introduction. How do scientific explanations generalize? When explanation is viewed as the application of scientific laws to specific cases, generalization is relatively straightforward: even if discovered in a particular instance, a regularity is rendered into a law by quantifying it universally. It then applies to any condition in which the antecedent is satisfied. But biologists, especially in domains such as cell and molecular biology, seldom invoke laws in their explanations. Instead, they seek to explain a phenomenon by uncovering and describing the biological mechanism that appears to be responsible for it. Several philosophical accounts of mechanistic explanation in biology have been advanced recently. Al-

$\dagger$ To contact the author, please write to: Department of Philosophy and Interdisciplinary Programs in Science Studies and Cognitive Science, 0119, University of California, San Diego, La Jolla, CA 92093-0119; e-mail: bill@mechanism.ucsd.edu.

\#I thank Adele Abrahamsen, Lindley Darden, and members of the University of California, San Diego, Philosophy of Biology Research Group and of the DC History and Philosophy of Biology Discussion Group for very helpful comments and suggestions.

Philosophy of Science, 76 (December 2009) pp. 762-773. 0031-8248/2009/7605-0003\$10.00 Copyright 2009 by the Philosophy of Science Association. All rights reserved. 
though the terminology varies somewhat across authors, the key elements of a mechanistic explanation are the identification of the relevant parts of the mechanism, the determination of the operations they perform, and an account of how the parts and operations are organized such that, under specific contextual conditions, the mechanism realizes the phenomenon of interest (Bechtel and Richardson 1993; Machamer, Darden, and Craver 2000; Bechtel and Abrahamsen 2005; Bechtel 2006; Darden 2006; Craver 2007). The challenge for understanding generalization is that a mechanistic account is highly particularized: researchers develop it for a model organism and study mechanisms in model organisms, and it is anticipated that there will be important differences - involving parts, operations, and organization-between already-studied organisms and those to which a scientist wishes to generalize a mechanistic explanation.

A first part of the answer to the question of how biologists generalize mechanistic explanations is that, as a result of descent from a common ancestor, biologists expect similarities among the mechanisms responsible for the same or similar phenomena in related organisms. Thus, biologists provisionally assume that the parts, operations, and organization of a mechanism in an ancestral species are largely conserved in descendant species. However, evolutionary processes also introduce variation; hence, researchers must probe for differences in parts, operations, and organization. This appeal to conservation is particularly productive when the mechanism is found to be largely conserved, as posited, but exhibits interesting variations. An altered operation may have resulted in other changes, for example, and the original account can guide researchers toward the most likely loci of such changes.

In this article I will illustrate how the assumption of conservation served both generalization and discovery in a particular case: research on circadian oscillators within animals. Circadian rhythms, the approximately 24-hour rhythms that are endogenously maintained, are widespread in living organisms and affect such aspects as body temperature, metabolism, endocrine function, locomotion, sexual activity, and mental function. Since circadian rhythms are found not only in organisms with a central nervous system but also in single-cell organisms, researchers generally assumed that the internal clock (oscillator) employed intracellular processes, even in higher organisms. In Drosophila, researchers focused on lateral neurons, whereas in mammals they concentrated on a structure in the hypothalamus above the optic chiasm known as the suprachiasmatic nucleus ( $\mathrm{SCN}$ ). The challenge was to explain how concentrations of certain proteins in these structures could oscillate with a period of approximately 24 hours. In the next sections, I consider how researchers first addressed this challenge using Drosophila as the model organism and how the as- 
sumption that the mechanism was conserved then led to a productive interaction with researchers studying mammals.

2. The Drosophila Model System for Studying Circadian Oscillators. Before the quest to identify the responsible mechanism, Drosophila had provided a model for studying circadian rhythms at the behavioral level due to the fact that fruit flies emerge from the pupa (eclose) only around dawn. Whatever time of day a fruit fly completes its development, it delays eclosing until the subsequent dawn. Even if the pupa are kept in total darkness, they eclose at what would have been dawn (Pittendrigh 1954). Fruit flies had also served as a model species for genetic research, so it is not surprising that Konopka and Benzer (1971) employed them to begin identifying genes affecting circadian behavior. Applying mutagens targeted to the X chromosome of Drosophila, they generated mutant flies that exhibited shortened, lengthened, and null oscillations in timing of eclosion. They traced all three mutations to a common locus and named the affected gene period (per).

The cloning of per in the mid-1980s by Michael Rosbash and his colleagues made it possible to fill in additional components of the molecular mechanism responsible for circadian rhythms. The expression of per results in increased concentrations of per messenger RNA (mRNA) and, in turn, of the protein PERIOD (PER) that is synthesized from per mRNA in the cytoplasm. (Protein names are standardly capitalized, while the names of genes are written in italics. In Drosophila the names of genes are entirely in lowercase, whereas in mammals the first letter is capitalized.) Hardin, Hall, and Rosbash (1990) determined that the concentrations of both per mRNA and PER exhibited circadian oscillations, with the peaks and valleys in PER concentrations following those of per mRNA by approximately 8 hours. Moreover, these cycles were shortened, lengthened, or absent in the previously discovered per mutants. Since PER is found not only in the cytoplasm but also in the nucleus, Hardin et al. proposed a feedback mechanism in which PER could inhibit the transcription of its own gene, per, as illustrated in Figure 1. When there is a low concentration of PER in the nucleus, the synthesis of additional molecules of PER proceeds normally in the cytoplasm. As they accumulate, they get transported back into the nucleus, where they inhibit further synthesis. But they break down over time and, failing to be replenished, no longer inhibit synthesis. The rate of synthesis returns to normal, beginning a new cycle. Appropriate timing of these operations results in a regular oscillation in PER concentrations, with the rise and fall (1 period) occupying about 24 hours.

This proposal, though, raised a number of questions about exactly how the feedback loop might operate. Particularly important were the ques- 


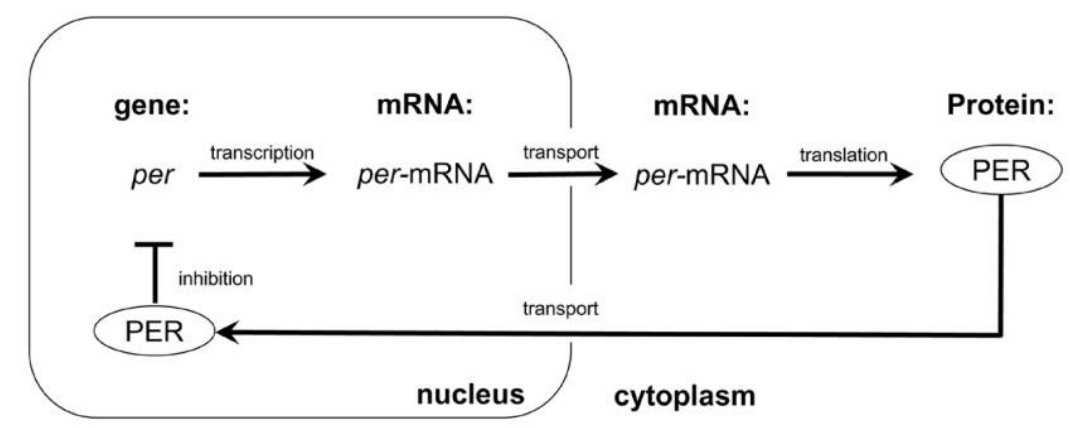

Figure 1. Proposed feedback mechanism for generating circadian oscillations.

tions of what delayed PER's transport back into the nucleus and how PER could inhibit per transcription. Researchers realized PER could not do this directly as it lacked a site where it could bind to DNA. This led researchers to search for other component parts in the clock mechanism.

A second component part of the mechanism was discovered by Sehgal et al. (1994). They followed essentially the same approach as Konopka, generating a large number of mutations involving the second and third chromosomes in Drosophila. One such mutation eliminated rhythmic eclosion or locomotion. In flies with this mutation, moreover, per mRNA concentrations ceased to oscillate. Seghal et al. took this to indicate an interaction between per and the new gene, which they named timeless (tim). A second study (Vosshall et al. 1994) indicated that tim is required for PER to be transported from the cytoplasm, where it is synthesized, back into the nucleus, where it can inhibit transcription of its own gene, and proposed that "PER contains sequences that somehow inhibit PER nuclear localization in the absence of tim" (1607). These researchers soon determined that PER and TIM form a dimer (a compound of two similar units) before both are transported back into the nucleus and that a region found on both PER and TIM, which they named the cytoplasmic localization domain, was responsible for preventing either alone from migrating into the nucleus (Gekakis et al. 1995). This region was presumably masked in the dimer, allowing the dimer to be transported into the nucleus (Saez and Young 1996).

The discovery of tim helped explain the translocation of PER into the nucleus, but it did not explain how PER (or TIM) inhibited its own transcription since TIM, like PER, lacked a DNA binding site. Investigators next explored the possibility that PER bound with a per activating factor and, when it did so, blocked the activator from activating per transcription. Support for the existence of such a factor was provided by 
the identification of an E-box (CACGTG) promoter upstream of per that is a target for a basic-helix-loop-helix (bHLH) DNA binding site on an activator protein and by determination that this E-box was required for robust cycling (Hao, Allen, and Hardin 1997). The activating protein, however, was first discovered not in Drosophila but in mammals.

3. Linking the Clocks in Drosophila and Mice. In the early 1990s the search for circadian mutants was also proceeding for mammals, especially mice. Vitaterna et al. (1994) identified a mutation that yielded increased period length and, in a homozygotic form, loss of rhythms after 2 weeks. They labeled the mutant gene Clock (for circadian locomotor output cycles kaput), localized it to chromosome 5, and determined that its protein oscillated in a circadian fashion. Three years later the same laboratory (King et al. 1997) cloned Clock and predicted "that this candidate gene encodes a novel member of the bHLH-PAS domain family of transcription factors" (645). Noting the assumption of Drosophila researchers that unknown transcription factors interact with PER, King et al. proposed that "the mouse Clock gene could be the mammalian ortholog of such a gene" (649). In addition, Gekakis et al. (1998) predicted that CLOCK must dimerize with a partner and identified BMAL1, a protein whose function was then unknown, as a protein whose concentrations oscillated in a similar manner as CLOCK. They also demonstrated that the mammalian CLOCK-BMAL1 dimer would bind with per's E-box in Drosophila and proposed processes by which PER might interfere with the action of CLOCK-BMAL1 to inhibit per expression.

The discovery of Clock in mice led Drosophila researchers to seek a Drosophila homolog, and they soon found a gene that possessed very similar bHLH and PAS domains (Darlington et al. 1998). Moreover, they determined that its protein, dCLOCK, was a specific activator of the per and tim promoters. They also found evidence for a Drosophila homolog of Bmall and concluded, "It is tempting to speculate that the Drosophila four-component transcriptional feedback loop described here is sufficient to generate a rudimentary circadian rhythm" (1602).

As this work was proceeding, other researchers followed up on an indication of a mammalian homolog to per and in 1997 identified such a homolog in mice and in humans. Examining the proteins, researchers found the mouse protein to be approximately $44 \%$ identical to the fly protein, with many of the differences involving neutral amino acid substitutions (Sun et al. 1997; Tei et al. 1997). Soon after, it was recognized that in fact there are multiple mammalian homologs of per, designated mPer 1, mPer2 (Albrecht et al. 1997), and mPer3 (Zylka et al. 1998). They reside on different chromosomes and differ in such respects as their responses to light pulses. One of the first noted consequences of the dif- 
ferentiation of three Per genes in mammals is increased robustness: deletion of just one Per gene does not eliminate circadian cycling, whereas deletion of both Perl and Per2 does.

4. Just How Conserved Is the Mammalian Clock? At this point there seemed to be a high degree of conservation between the Drosophila and mammalian clock mechanism. Mammalian homologs had been found for per, clock, and bmall; the only major change was that per had differentiated into three mammalian genes. This supported a straightforward generalization of the mechanistic account achieved for Drosophila to mammals. But the story soon became more complex as researchers sought a mammalian homolog for the fourth component of the Drosophila clock, tim. They found such a gene: its protein occurs in the SCN and can dimerize with mPER 1 or mPER 2 as well as with dPER. In Drosophila cells the dimers are transported back into the nucleus and inhibit per transcription; in mouse cells the TIM : mPER 1 inhibited activation of the mPerl promoter (Sangoram et al. 1998). So far, the parallels held up. But there were significant differences: neither the concentrations of mTim mRNA nor its protein, mTIM, oscillate under constant light or darkness, and in response to light mTIM levels increase whereas dTIM levels decrease.

These seemingly minor differences took on greater significance in the context of a major feature of circadian rhythms, their entrainment (resetting) by exposure to light, especially around subjective dawn or subjective dusk. This process is essential for organisms to adjust to seasonal differences or for us to adjust after travel to different time zones. The discovery of photoreceptors in Drosophila that are conserved from bluelight photoreceptors (known as cryptochromes) in plants provided a critical clue to the entrainment mechanism. Searching for mammalian homologs to cry, Todo et al. (1996) found two each in mice and humans. Unlike in plants, concentrations of cryptochromes in Drosophila and in mammals were found to undergo circadian oscillations, indicating they might have significant clock functions. In Drosophila, Emery et al. (1998) determined that this oscillation was due to light exposure, by showing that when flies were maintained in continuous darkness, CRY levels did not oscillate but rather continued to increase. They inferred that "CRY is a major photoreceptor for Drosophila locomotor activity rhythms" (674). Since TIM concentrations are responsive to CRY levels in wild-type flies but not in $c r y^{b}$ mutants (Stanewsky et al. 1998) and CRY light regulation is not affected by TIM or other clock molecules, investigators concluded that CRY served an entrainment function by affecting concentrations of TIM.

Assuming conservation, researchers at first thought that CRY figured in the entrainment pathway in mammals as well, and the initial evidence 
lent support. Miyamoto and Sancar (1998) found that CRY1 and CRY2 occur in retinal ganglion cells as well as the inner nuclear layer of the mouse retina and that CRY1 concentrations oscillate in a circadian manner in the SCN itself. They interpreted this evidence as indicating a photoreceptor role for both CRY proteins. Thresher et al. (1998) offered further support for this view by showing that CRY2-deficient mutants failed to entrain to light. Thresher et al. also observed, however, that CRY2-deficient mutants showed elongated periods in total darkness, a finding that would not be expected if CRY2 figured only in entrainment. Whereas Thresher et al. attempted to explain away this finding, other evidence soon pointed to a different conclusion - that mammalian CRY homologs were not serving the entrainment function and were actually part of the central clock mechanism. One piece of evidence was the finding that loss of both CRY1 and CRY2 eliminated circadian rhythms in constant darkness and loss of just one resulted in shortened (CRY1) or lengthened (CRY2) periods of oscillation (van der Horst et al. 1999). Using an assay in cultured cells in which CLOCK-BMAL1 drives a luciferase reporter gene from the mPerl gene E-box, Griffin, Staknis, and Weitz (1999) determined that in humans both CRY1 and CRY2 produced a specific inhibition of CLOCK-BMAL1 activity. This indicated that the "role for CRYs in the mammalian circadian clock is to inhibit Perl gene expression" (769). Citing as well evidence that CRY no longer performed an entrainment function, these researchers concluded that CRY1 and CRY2 had supplanted TIM in the mammalian clock. (Figure 2 shows this change as well as the role of each as a dimerization partner with PER.)

The discovery that, although conserved, mCRYs were no longer operative in entrainment in mammals generated a new research question: What performs the entrainment operation in mammals? The clue was again provided by conservation. Melanopsin, a member of the opsin family of photopigments, was discovered in melanophores (melanin pigment containing cells) of the frog Xenopus laevis (Provencio et al. 1998). Subsequently, Provencio et al. (2000) reported finding melanopsin in the mammalian inner retina. Linking melanopsin to circadian entrainment required demonstrating its presence in the retinohypothalamic tract that had long been known to provide the input to the SCN. Hannibal had previously identified pituitary adenylate cyclase activating peptide (PACAP) as the neurotransmitter active in the retinohypothalamic tract during subjective day, and he now showed that both melanopsin mRNA and protein are found in the same cells as PACAP (Hannibal 2002). Although knockout of melanopsin alone does not eliminate entrainment, it does reduce it (Ruby et al. 2002), and when the knockout is combined with loss of the rods and cones, entrainment is eliminated (Hattar et al. 2003). Together, 
Subjective Nighttime

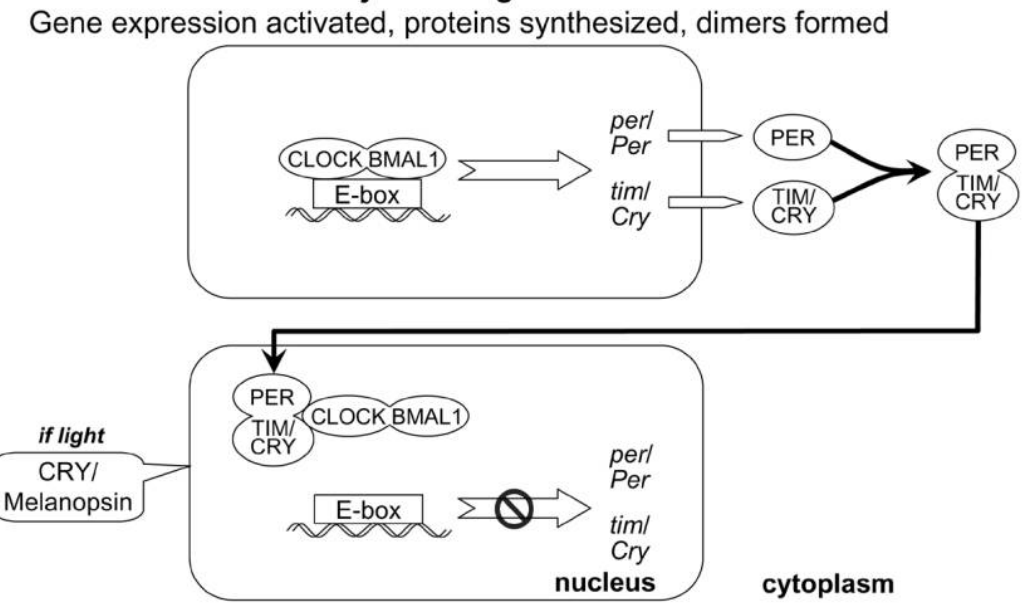

Subjective Daytime

Dimers enter nucleus, further gene expression supressed

Figure 2. Comparison of Drosophila and mammalian clocks. CRY replaces TIM as the dimerization partner of PER. PER/TIM and PER/CRY dimers interact with the BMAL1/CLOCK dimer, removing them as activators of per/Per and tim/Cry genes. Melanopsin replaces CRY in entraining the clock.

this provided strong evidence that melanopsin had assumed the entrainment function of CRY in mammals.

With melanopsin replacing CRY in entrainment, and CRY replacing TIM in the central oscillator, a natural question is, What happened to TIM? It is still expressed in mammals, but many researchers initially concluded it ceased to perform any clock function (Albrecht 2002). Griffin et al., in the study that suggested CRY had supplanted TIM, had described an antagonistic interaction between either CRY1 or CRY2 and TIM, which they took to "suggest cross-regulation among the proteins inhibiting CLOCK-BMAL1 activity within the circadian clock feedback loop" (1999, 470). Since deletion of mTim is lethal in embryonic development, the role of TIM could not be settled through a knockout experiment. The development of the gene knockdown technique in which a reagent complementary to an active gene (antisense oligodeoxynucleotides, $\alpha \mathrm{ODN}$ ) binds to the site and suppresses its expression allowed for a more specific examination of the role of mTim in slice preparations. When an $\alpha \mathrm{ODN}$ targeted to the sequence surrounding the start codon of mTim was applied for prolonged periods, full-length mTIM (mTIM-fl) expression was suppressed. Importantly, so were circadian rhythms (Barnes et al. 2003). 
When the $\alpha \mathrm{ODN}$ was administered for only 2 hours, it resulted in a phase shift if applied during subjective day (advances when applied early, delays when applied late) but not if administered during subjective night. It was also demonstrated that mTIM-fl interacts with the three mPERs as well as both mCRYs. In the knockdown preparation, levels of the mPERs decreased, a finding that corresponds well with the low levels of dPER in dtim mutants and, according to Barnes et al., shows that "mTIM-fl is a functional homolog of dTIM." Relying on additional evidence, Barnes et al. propose locating " $m$ Tim on the negative arm of the molecular feedback loop in the SCN, as it is in the Drosophila clock" (441). In particular, they hypothesized "that mPER2 may be the physiologically preferred partner of mTIM- $\mathrm{fl}$ and that this heterodimer could be the functional mammalian counterpart of dTIM-dPER. Addition of $m$ Tim to the mammalian clockwork completes a core having each functional homolog of the Drosophila clockwork and emphasizes the highly conserved nature of the biological timekeeping mechanism" (441). At present, the question of the role of Tim remains undecided.

5. Conservation as a Strategy for Both Generalization and Discovery. The initial success in developing a core mechanism for circadian oscillations in Drosophila invited the heuristic of seeking mammalian homologs for the Drosophila genes so as to generalize the explanation to mammals. This proved successful in the case of per in which three mammalian homologs were identified, at least two of which play comparable roles in the mammalian clock. Independent work on the mammalian clock resulted in the identification of two additional components of the clock mechanism, Clock and Bmall, and this guided the search for homologs in Drosophila. The assumption of conservation and the search for homologs thus serves as a heuristic for generalizing accounts of mechanisms across species.

Conservation provides the basis for an even more powerful discovery heuristic when the mechanism in the model organism does not align perfectly with the one in the species of interest. The quest for the mammalian homolog of cry, which figures centrally in the entrainment mechanism in Drosophila, led to the discovery that, in mammals, Cry appears not to figure in entrainment but rather to have usurped the role of tim. Initially this discovery was viewed as showing that Tim had no clock function in mammals, but the fact that Tim continues to be expressed motivated more detailed studies that have suggested that it might retain a clock function by serving as the preferred dimerization partner of one of the mammalian PER homologs. Discovering a change in the operation performed by CRY, however, also prompted a search for what filled the role that CRY played in Drosophila. This yielded the discovery of a different photopigment, 
melanopsin, and an investigation into whether it might figure in entrainment in mammals.

Scientific discovery was once viewed as an intractable problem for philosophers. Several philosophers who have addressed mechanistic explanation in biology have noted that the search for mechanisms is often guided by discovery heuristics (Bechtel and Richardson 1993; Darden and Craver 2002). The conservation of mechanisms further advances this project. Mechanisms are typically investigated in specific model organisms chosen because of their accessibility for study. These model organisms are often not the primary systems of interest. The assumption of conservation of component parts, operations, and organization undergirds a heuristic of seeking conserved components and generalizing from studied mechanisms to those of principal interest. Moreover, when conservation turns out not to be perfect, it gives rise to other discovery heuristics. When a component is preserved but appears to be performing a different operation, researchers are prompted to investigate what performs its old operation and what happens to the component that had performed the new operation.

\section{REFERENCES}

Albrecht, Urs (2002), "Functional Genomics of Sleep and Circadian Rhythm: Invited Review; Regulation of Mammalian Circadian Clock Genes", Journal of Applied Physiology 92: $1348-1355$.

$\rightarrow$ Albrecht, Urs, Zhong Sheng Sun, Gregor Eichele, and Cheng Chi Lee (1997), "A Differential Response of Two Putative Mammalian Circadian Regulators, mperl and mper2, to Light", Cell 91: 1055-1064.

$\rightarrow$ Barnes, Jessica W., Shelley A. Tischkau, Jeffrey A. Barnes, Jennifer W. Mitchell, Penny W. Burgoon, Jason R. Hickok, and Martha U. Gillette (2003), "Requirement of Mammalian Timeless for Circadian Rhythmicity", Science 302: 439-442.

Bechtel, William (2006), Discovering Cell Mechanisms: The Creation of Modern Cell Biology. Cambridge: Cambridge University Press.

$\rightarrow$ Bechtel, William, and Adele Abrahamsen (2005), "Explanation: A Mechanist Alternative", Studies in History and Philosophy of Biological and Biomedical Sciences 36: 421-441.

Bechtel, William, and Robert C. Richardson (1993), Discovering Complexity: Decomposition and Localization as Strategies in Scientific Research. Princeton, NJ: Princeton University Press.

Craver, Carl (2007), Explaining the Brain: What a Science of the Mind-Brain Could Be. New York: Oxford University Press.

Darden, Lindley (2006), Reasoning in Biological Discoveries: Essays on Mechanisms, Interfield Relations, and Anomaly Resolution. Cambridge: Cambridge University Press.

$\rightarrow$ Darden, Lindley, and Carl Craver (2002), "Strategies in the Interfield Discovery of the Mechanism of Protein Synthesis", Studies in History and Philosophy of Biological and Biomedical Sciences 33: 1-28.

$\rightarrow$ Darlington, Thomas K., Karen Wager-Smith, M. Fernanda Ceriani, David Staknis, Nicholas Gekakis, Thomas D. Steeves, Charles J. Weitz, Joseph S. Takahashi, and Steve A. Kay (1998), "Closing the Circadian Loop: Clock-Induced Transcription of Its Own Inhibitors per and tim", Science 280: 1599-1603.

$\rightarrow$ Emery, Patrick T., W. V. So, M. Kanedo, J. C. Hall, and Michael Rosbash (1998), "CRY, 
a Drosophila Clock and Light-Regulated Cryptochrome, Is a Major Contributor to Circadian Rhythm Resetting and Photosensitivity", Cell 95: 669-679.

$\rightarrow$ Gekakis, Nicholas, Lino Saez, Anne-Marie Delahaye-Brown, Michael P. Myers, Amita Sehgal, Michael W. Young, and Charles J. Weitz (1995), "Isolation of timeless by PER Protein Interaction: Defective Interaction between timeless Protein and Long-Period Mutant PER ${ }^{\mathrm{L}}$, Science 270: 811-815.

$\rightarrow$ Gekakis, Nicholas, David Staknis, Hubert B. Nguyen, Fred C. Davis, Lisa D. Wilsbacher, David P. King, Joseph S. Takahashi, and Charles J. Weitz (1998), "Role of the Clock Protein in the Mammalian Circadian Mechanism", Science 280: 1564-1569.

$\rightarrow$ Griffin, Edmund A., Jr., David Staknis, and Charles J. Weitz (1999), "Light-Independent Role of CRY1 and CRY2 in the Mammalian Circadian Clock", Science 286: 768-771.

$\rightarrow$ Hannibal, Jens (2002), "Neurotransmitters of the Retino-Hypothalamic Tract", Cell and Tissue Research 309: 73-88.

Hao, H., D. L. Allen, and Paul E. Hardin (1997), "A Circadian Enhancer Mediates PERDependent mRNA Cycling in Drosophila Melanogaster", Molecular Cell Biology 17: 3687-3693.

$\rightarrow$ Hardin, Paul E., Jeffrey C. Hall, and Michael Rosbash (1990), "Feedback of the Drosophila period Gene Product on Circadian Cycling of Its Messenger RNA Levels", Nature 343: 536-540.

$\rightarrow$ Hattar, Samer, Robert J. Lucas, N. Mrosovsky, S. Thompson, R. H. Douglas, Mark W. Hankins, J. Lem, M. Biel, F. Hofmann, Russell G. Foster, and King-Wai Yau (2003), "Melanopsin and Rod-Cone Photoreceptive Systems Account for All Major Accessory Visual Functions in Mice", Nature 424: 75-81.

$\rightarrow$ King, David P., Yaliang Zhao, Ashvin M. Sangoram, Lisa D. Wilsbacher, Minoru Tanaka, Marina P. Antoch, Thomas D. L. Steeves, Martha Hotz Vitaterna, Jon M. Kornhauser, Phillip L. Lowrey, Fred W. Turek, and Joseph S. Takahashi (1997), "Positional Cloning of the Mouse Circadian Clock Gene", Cell 89: 641-653.

Konopka, Ronald J., and Seymour Benzer (1971), "Clock Mutants of Drosophila Melanogaster", Proceedings of the National Academy of Sciences of the United States of America 89: 2112-2116.

$\rightarrow$ Machamer, Peter, Lindley Darden, and Carl Craver (2000), "Thinking about Mechanisms", Philosophy of Science 67: 1-25.

$\rightarrow$ Miyamoto, Yasuhide, and Aziz Sancar (1998), "Vitamin B2-Based Blue-Light Photoreceptors in the Retinohypothalamic Tract as the Photoactive Pigments for Setting the Circadian Clock in Mammals", Proceedings of the National Academy of Sciences of the United States of America 95: 6097-6102.

$\rightarrow$ Pittendrigh, Colin S. (1954), "On Temperature Independence in the Clock-System Controlling Emergence Time in Drosophila", Proceedings of the National Academy of Sciences of the United States of America 40: 1018-1029.

$\rightarrow$ Provencio, Ignacio, Guisen Jiang, Willem J. De Grip, William Par Hayes, and Mark D. Rollag (1998), "Melanopsin: An Opsin in Melanophores, Brain, and Eye", Proceedings of the National Academy of Sciences of the United States of America 95: 340-345.

Provencio, Ignacio, Ignacio R. Rodriguez, Guisen Jiang, William Par Hayes, Ernesto F. Moreira, and Mark D. Rollag (2000), "A Novel Human Opsin in the Inner Retina", Journal of Neuroscience 20: 600-605.

$\rightarrow$ Ruby, Norman F., Thomas J. Brennan, Xinmin Xie, Vinh Cao, Paul Franken, H. Craig Heller, and Bruce F. O'Hara (2002), "Role of Melanopsin in Circadian Responses to Light", Science 298: 2211-2213.

$\rightarrow$ Saez, Lino, and Michael W. Young (1996), "Regulation of Nuclear Entry of the DrosophilaClock Proteins Period and Timeless", Neuron 17: 979-990.

$\rightarrow$ Sangoram, Ashvin M., Lino Saez, Marina P. Antoch, Nicholas Gekakis, David Staknis, Andrew Whiteley, Ethan M. Fruechte, Martha Hotz Vitaterna, Kazuhiro Shimomura, David P. King, Michael W. Young, Charles J. Weitz, and Joseph S. Takahashi (1998), "Mammalian Circadian Autoregulatory Loop: A Timeless Ortholog and Mperl Interact and Negatively Regulate CLOCK-BMAL1-Induced Transcription", Neuron 21: 11011113 .

$\rightarrow$ Sehgal, Amita, Jeffrey L. Price, Bernice Man, and Michael W. Young (1994), "Loss of 
Circadian Behavioral Rhythms and per RNA Oscillations in the Drosophila Mutant timeless", Science 263: 1603-1606.

$\rightarrow$ Stanewsky, Ralf, Maki Kaneko, Patrick Emery, Bonnie Beretta, Karen Wager-Smith, Steve A. Kay, Michael Rosbash, and Jeffrey C. Hall (1998), "The $c r y^{b}$ Mutation Identifies Cryptochrome as a Circadian Photoreceptor in Drosophila", Cell 95: 681-692.

$\rightarrow$ Sun, Zhong Sheng, Urs Albrecht, Olga Zhuchenko, Jennifer Bailey, Gregor Eichele, and Cheng Chi Lee (1997), "RIGUI, a Putative Mammalian Ortholog of the Drosophila period Gene", Cell 90: 1003-1011.

$\rightarrow$ Tei, Hajime, Hitoshi Okamura, Yasufumi Shigeyoshi, Chiaki Fukuhara, Ritsuko Ozawa, Matsumi Hirose, and Yoshiyuki Sakaki (1997), "Circadian Oscillation of a Mammalian Homologue of the Drosophila period Gene", Nature 389: 512-516.

$\rightarrow$ Thresher, Randy J., Martha Hotz Vitaterna, Yasuhide Miyamoto, Aleksey Kazantsev, David S. Hsu, Claude Petit, Christopher P. Selby, Lale Dawut, Oliver Smithies, Joseph S. Takahashi, and Aziz Sancar (1998), "Role of Mouse Cryptochrome Blue-Light Photoreceptor in Circadian Photoresponses", Science 282: 1490-1494.

$\rightarrow$ Todo, Takeshi, Haruko Ryo, Kazuo Yamamoto, Hiroyuki Toh, Taiichiro Inui, Hitoshi Ayaki, Taisei Nomura, and Mituo Ikenaga (1996), "Similarity among the Drosophila (6-4) Photolyase, a Human Photolyase Homolog, and the DNA Photolyase-Blue-Light Photoreceptor Family", Science 272: 109-112.

$\rightarrow$ van der Horst, Gijsbertus T. J., Manja Muijtjens, Kumiko Kobayashi, Riya Takano, Shinichiro Kanno, Masashi Takao, Jan de Wit, Anton Verkerk, Andre P. M. Eker, Dik van Leenen, Ruud Buijs, Dirk Bootsma, Jan H. J. Hoeijmakers, and Akira Yasui (1999), "Mammalian Cry1 and Cry2 Are Essential for Maintenance of Circadian Rhythms", Nature 398: 627-630.

$\rightarrow$ Vitaterna, Martha Hotz, David P. King, Anne-Marie Chang, Jon M. Kornhauser, Phillip L. Lowrey, J. David McDonald, William F. Dove, Lawrence H. Pinto, Fred W. Turek, and Joseph S. Takahashi (1994), "Mutagenesis and Mapping of a Mouse Gene, Clock, Essential for Circadian Behavior", Science 264: 719-725.

$\rightarrow$ Vosshall, Leslie B., Jeffrey L. Price, Amita Sehgal, Lino Saez, and Michael W. Young (1994), "Block in Nuclear Localization of period Protein by a Second Clock Mutation, timeless", Science 263: 1606-1609.

$\rightarrow$ Zylka, Mark J., Lauren P. Shearman, David R. Weaver, and Steven M. Reppert (1998), "Three period Homologs in Mammals: Differential Light Responses in the Suprachiasmatic Circadian Clock and Oscillating Transcripts Outside of Brain”, Neuron 20: 11031110. 